\title{
Clinical efficacy of the enzyme replacement therapy in patients with late-onset Pompe disease: a systematic review and a meta-analysis
}

\author{
Berli Sarah $^{1} \cdot$ Brandi Giovanna $^{1} \cdot$ Keller Emanuela ${ }^{1,2} \cdot$ Najia Nadi $^{1} \cdot$ Vitale Josè $^{3,4} \cdot$ Pagnamenta Alberto $^{3,5,6}$
}

Received: 12 February 2021 / Revised: 16 March 2021 / Accepted: 18 March 2021 / Published online: 13 April 2021

(c) The Author(s) 2021

\begin{abstract}
In patients with late-onset Pompe disease (LOPD), the efficacy of the enzyme replacement therapy (ERT) with recombinant human alpha-glucosidase (rhGAA) is difficult to evaluate, due to the clinical heterogeneity and the small sample sizes in published studies. Therefore, we conduct a systematic literature review and meta-analysis of the literature to evaluate the efficacy of ERT in LOPD patients considering the walking distance, respiratory function and muscle strength. Particularly, six-minute walk test (6MWT), forced vital capacity (FVC), medical research council (MRC) grading, quantitative muscle testing (QMT), and quick motor function test (QMFT) were outcomes of interest. Overall, 619 studies were identified in PubMed, EMBASE and by manual search on July 18th, 2020. After an initial assessment, 16 studies were included in the meta-analysis, containing clinical data from 589 patients with LOPD. For the 6MWT, 419 patients were analyzed. Walking distance improved on average, $32.2 \mathrm{~m}$ greater during the observed period $(p=0.0003)$, compared to the distance at the baseline. The meta-analysis did not show any improvement in FVC and only a tendency towards better muscle strength after treatment with ERT, but the difference was not statistically significant. In conclusion, the available data showed that ERT has a significant beneficial efficacy in the improvement of walking distance in LOPD patients and a non-significant improvement of muscle strength. No improvement in respiratory capacity was found. More prospective and controlled trials are needed to demonstrate a clear clinical benefit of ERT.
\end{abstract}

Keywords Pompe disease · Gycogen storage disease type II - Late-onset Pompe disease · Enzyme replacement therapy · Recombinant human alpha-glucosidase $\cdot$ Systematic review $\cdot$ Meta-analyse

Berli Sarah and Brandi Giovanna have contributed equally to this work.

Brandi Giovanna

giovanna.brandi@usz.ch

1 Institute for Intensive Care Medicine, University Hospital Zurich, Rämistrasse 100, 8091 Zurich, Switzerland

2 Department of Neurosurgery and Clinical Neuroscience Center, University Hospital and University of Zurich, Zurich, Switzerland

3 Intensive Care Unit, Regional Hospital Mendrisio, Ente Ospedaliero Cantonale, Bellinzona, Switzerland

4 Centro Medico, Mendrisio, Switzerland

5 Unit of Biostatistics, Ente Ospedaliero Cantonale, Bellinzona, Switzerland

6 Division of Pneumology, University Hospital of Geneva, Geneva, Switzerland

\section{Introduction}

Pompe disease (PD) is a rare, inheritable, multisystemic lysosomal storage disorder caused by a deficiency of acid alpha-glucosidase (GAA), which leads to accumulation of glycogen within lysosomes, especially in the heart, skeletal and smooth muscles, and in the nervous system [1,2]. Two forms of PD-infantile-onset (IOPD) and late-onset (LOPD) - are classically recognized. Depending on the age of symptoms' onset, LOPD is sub-classified in a juvenile (JOPD) and in an adult form [3, 4]. IOPD is the most severe form and is characterized by cardiomegaly and generalized muscle weakness presenting during the first few months of life. LOPD, indeed, is clinically more heterogeneous [5], involving primarily skeletal and respiratory muscles leading to proximal weakness and respiratory insufficiency typically without cardiac manifestations [2,5]. Respiratory failure 
represents the leading cause of death in LOPD patients $[1$, $6,7]$.

In 2006 the first specific enzyme replacement therapy (ERT) with recombinant human GAA (rhGAA) was approved for PD treatment in Europe and the US [8] with the recommended dose of $20 \mathrm{mg} / \mathrm{kg}$ via IV infusion biweekly [6]. In IOPD patients, previous ERT studies already demonstrated prolonged long-term survival, improved cardiac function, and a decreased need for respiratory support [9]. In patients with LOPD, on the other hand, it is difficult to assess the general long-term effects of ERT on disease progression from individual studies due to a wide range of phenotypic presentations, the very low prevalence of the disease, and the small sample sizes in published studies. Systematic literature reviews assessing the impact of ERT in LOPD patients were previously conducted [10-12]. Toscano et al. found that at least two-thirds of treated patients showed improvement or stabilization of muscular and respiratory function after initiation of ERT [10]. Schoser et al. reported in a systematic review and meta-analysis a nearly five-fold lower mortality rate in treated patients than untreated patients and an improved respiratory function within the first few months followed by a slow return to baseline and an improved muscular function, which remains sustained over time [11]. By the consensus meetings of the European Pompe Consortium, a systematic review with a narrative synthesis due to the expected heterogeneity was undertaken showing a beneficial effect of ERT at the group level but with varied treatment effects at the individual level [12]. For JOPD patients only, a systematic literature review without statistical synthesis due to a low quality of original articles suggested a short-term benefit from ERT through improved muscle strength and reduced need for assisted ventilation [13].

The present work provides an important update on the current evidence on the clinical efficacy of ERT in patients with LOPD with regard to motor performance, respiratory function, and muscle strength compared to their baseline values. A systematic literature review with meta-analysis was conducted to assess the efficacy of treating LOPD with ERT on six-minute walk test (6MWT), forced vital capacity (FVC), and muscle strength measured with medical research council (MRC), quantitative muscle testing (QMT) and quick motor function test (QMFT).

\section{Material and methods}

PRISMA guidelines (Preferred Reporting of Items in Systematic Reviews and Meta-analyses) [14] were employed to guide review processes.

This systematic literature review was registered at the international prospective register of systematic reviews (PROSPERO; registration number: CRD42020182462).

\section{Search strategy}

Studies were identified with the MEDLINE (1966 to July 18th, 2020) and EMBASE (1980 to July 18th 2020) electronic databases. The search strategy was developed with the following keywords: Pompe disease, alpha glucosidase deficiency, acid maltase deficiency, glycogen storage disease type 2, enzymatic replacement therapy, enzyme replacement therapy, forced vital capacity, FVC, respiratory function, six-minute walk test, 6MWT, medical research council, $M R C$, quantitative muscle testing, QMT, quick motor function test, QMFT. The electronic search was supplemented by a manual search of reference lists and recent reviews, and by reviewing abstract books from the congress abstracts from the annual meetings.

Two reviewers (SB and GB) independently screened titles and abstracts and duplicates. Secondly, they screened the corresponding publications in full text to assess if the studies met the inclusion criteria. All disagreements were resolved by discussion between the two reviewers. All persisting disagreements were reviewed and resolved by a third conciliator $(\mathrm{NN})$. The final inclusion of studies was based on the agreement of all reviewers.

\section{Inclusion and exclusion criteria}

The study selection criteria are presented in Table 1 using the PICOS (Population, Interventions, Comparisons, Outcomes, and Study design) acronym.

All identified findings were reported using a flow chart according to PRISMA statement (Fig. 1).

Randomized controlled trials (RCTs), prospective observational studies, and retrospective studies with more than ten patients that evaluated rhGAA effects in patients with LOPD were eligible.

Case reports, case series with less than ten subjects, studies in patients with IOPD, experimental studies, abstracts, studies without baseline data or control group, and reports with no values in the results were excluded. The search was limited to articles written in English.

\section{Data extraction}

The extracted data were: trial's characteristics (first author, publication year), kind of study, treatment doses, disease duration at the start of ERT (from symptom onset), control group, selected outcome results baseline and after treatment begin with ERT (value, difference, P-value) and follow-up duration with ERT. Information was summarized using a pre-defined Excel Table.

Outcomes of interest were: motor performance, as assessed by the 6MWT; respiratory function, by considering 
Table 1 Inclusion criteria: Scope of the literature review in PICOS form

\begin{tabular}{ll}
\hline Criteria & Definition \\
\hline Population & Patients with LOPD \\
Interventions & Recombinant human GAA $20 \mathrm{mg} / \mathrm{kg}$ every two weeks \\
Comparison & Patients baseline values \\
Outcomes & 6-min walk test (6MWT) \\
& Forced vital capacity (FVC) \\
& Muscle strength: medical research council (MRC) grading, \\
& quantitative muscle testing (QMT), quick motor function test \\
& (QMFT) \\
Study Design & RCTs \\
& Open-label extension phases of included RCTs \\
& Single-arm trials \\
& Prospective observational studies \\
Retrospective studies with more than 10 patients
\end{tabular}

Fig. 1 Study selection Flow diagram
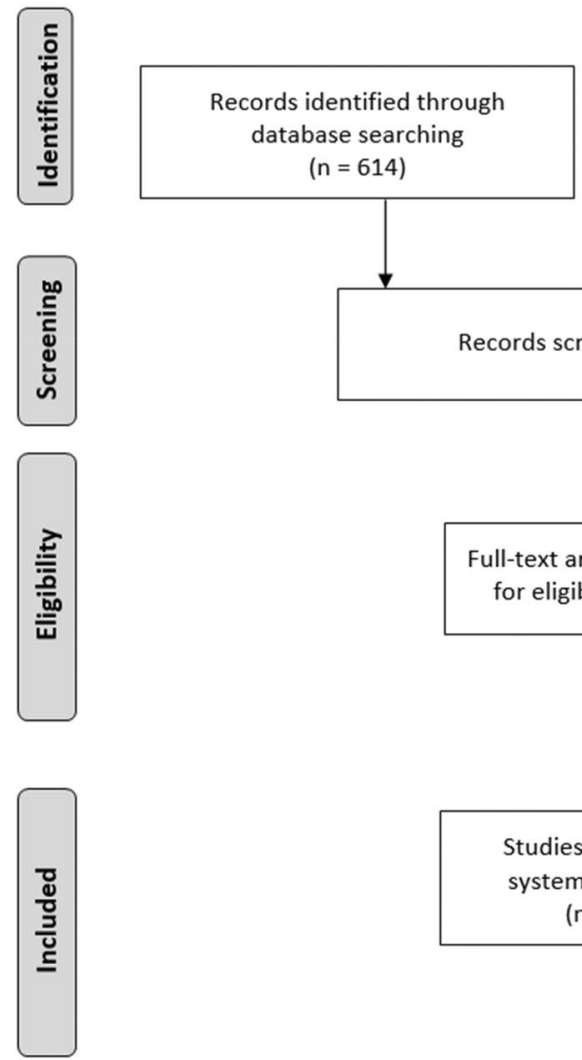

$$
(n=614)
$$

Additional records identified through other sources $(n=5)$

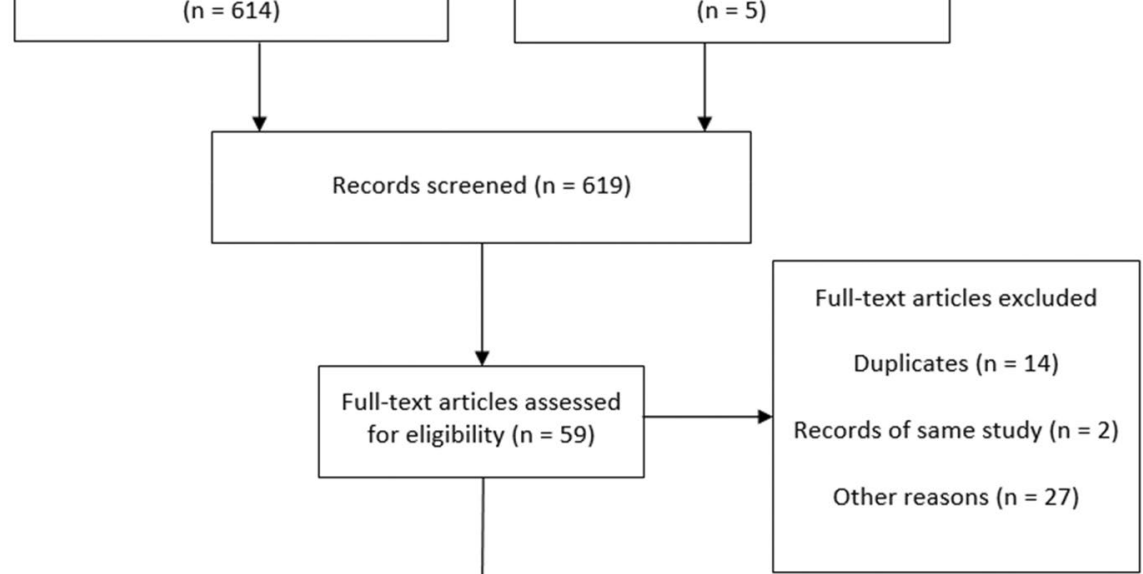

Studies included in systematic review $(n=16)$

the FVC; and muscle strength, as tested with the MRC grading scale, QMT and QMFT.

\section{Statistical analysis}

For each specific outcome (motor performance, respiratory function and muscle strength) the effect estimate and its corresponding 95\%-confidence interval (95\%-CI) were calculated for each study separately according to the inverse-variance fixed-effect method [15]. Each trial-specific effect size was subsequently combined across studies to calculate summary estimates and presented as a forest plot. Effect size on motor function was assessed on mean difference in 6MWT, effect size on respiratory function was evaluated on mean difference in FVC, whereas effect size on muscle strength was assessed on the standardized mean difference. The test statistic $Q$ was applied to assess the statistical evidence of heterogeneity. Due to the conservative nature 
of the test a cut-off of $p<0.1$ was used. For the estimation of the degree of heterogeneity $I^{2}$ statistic was used. The presence of publication bias was explored with a funnel plot. All analyses were performed using RevMan 5.3 (Review Manager (RevMan) [Computer program]. Version 5.3. Copenhagen: The Nordic Cochrane Centre, The Cochrane Collaboration, 2014).

\section{Results}

The systematic search identified 614 studies in the databases PubMed and EMBASE. Through manual search five additional papers were identified. Overall, 619 abstracts were considered as potentially eligible. After screening based on the inclusion criteria, 59 were selected for full-text review, as shown in the flow diagram of Fig. 1. Finally, a total of 16 studies were included in the meta-analysis, containing clinical data from 589 patients with LOPD [1, 7, 16-29].

The list and characteristics of the included papers are presented in Table 2: 6-min walk test (6MWT); forced vital capacity (FVC); and muscle strength (MRC), (QMT) and (QMFT).

The included studies were published between 2010 and 2020.

\section{Motor performance: 6-min walk test}

Fourteen studies, including 419 patients with LOPD (of those, 407 were treated with rhGAA) reported results concerning 6MWT as shown in Fig. 2 [1, 7, 16-19, 21-23, 25-29]. Follow-up time varied from 6 months to 7 years.

Forest plot of stratified analysis demonstrated that after 18.75 months, patients on rhGAA showed significant improvements in walking distance that were, on average, approximately $32.22 \mathrm{~m}$ (95\%-CI $14.98-49.47 ; p=0.0003)$ greater during the observed period, compared to the distance at baseline time.

Assessment of publication bias using a funnel plot indicated symmetry as shown in Fig. 3.

\section{Respiratory function: forced vital capacity}

Sixteen studies, including 563 patients with LOPD (of those, 555 were treated with rhGAA) reported results concerning (FVC) as shown in Fig. 4 [1, 7, 16-29].

Follow-up time varied from 6 months to 7 years.

Forest plot of stratified analysis demonstrated that after 20.75 months, patients on rhGAA did not show significant changes. FVC remained stable with approximately $-0.01 \%$ (95\%-CI $-2.48-2.45 ; p=0.99)$ during the observed period, compared to the FVC at the baseline time.

Table 2 Characteristics of the included papers

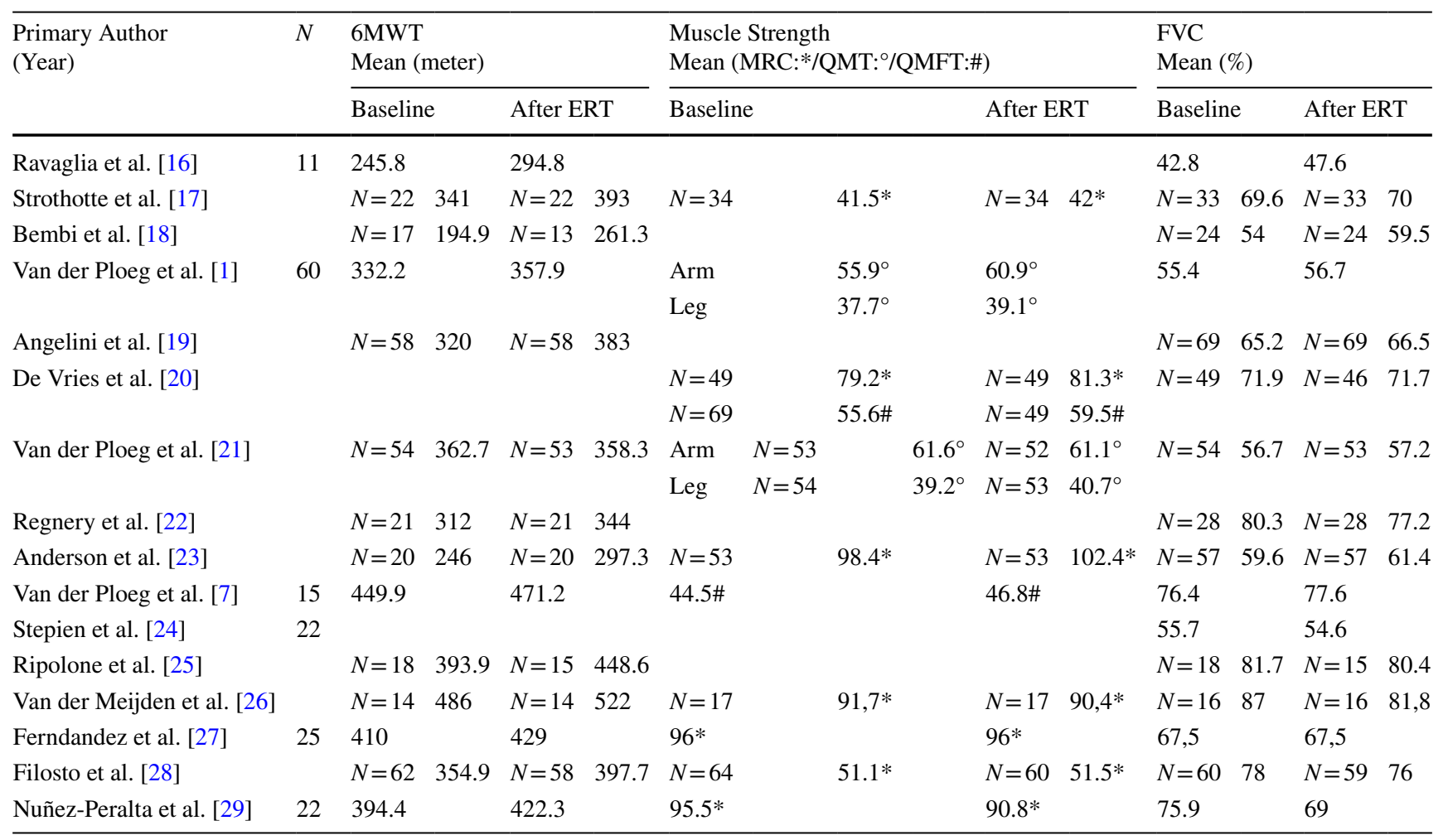




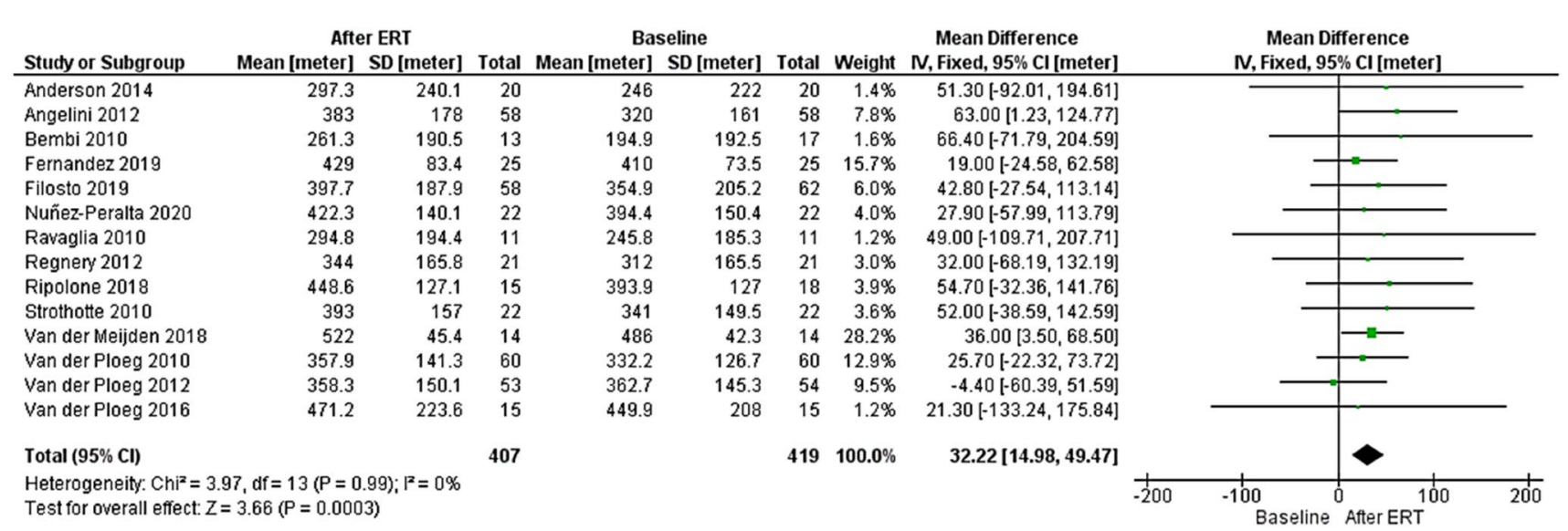

Fig. 2 Forest plot of effect of enzyme replacement therapy on motor performance, as assessed by 6-min-walk test

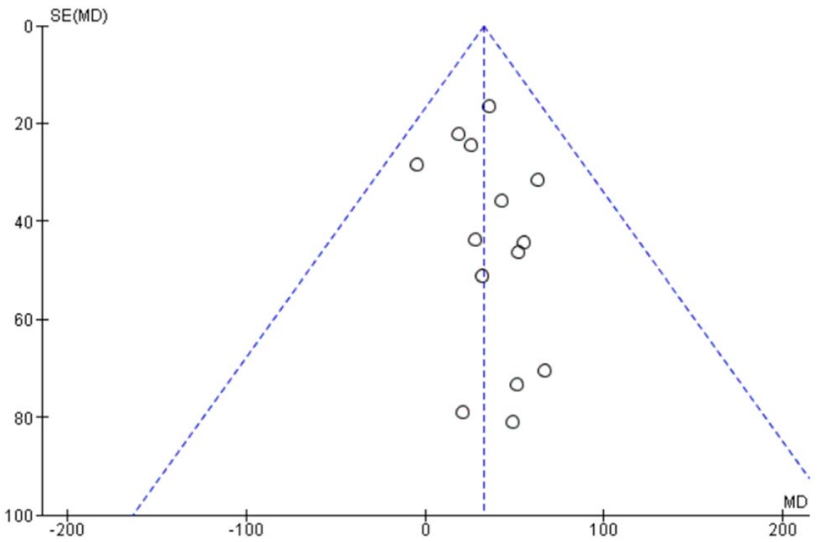

Assessment of publication bias using a funnel plot indicated symmetry as shown in Fig. 5.

\section{Muscle strength: medical research council grading scale, quantitative muscles testing and quick motor function test}

Ten studies, including 413 patients with LOPD (of those, 388 were treated with rhGAA) reported 575 measurements of muscle strength at baselines and 549 measurements after ERT assessed with MRC, QMT or QMFT as shown in Fig. 6 $[1,7,16,17,19-24,26-29]$.

Follow-up time varied from 6 months to 7 years.

Fig. 3 Funnel plot of comparison of 6-min-walk test

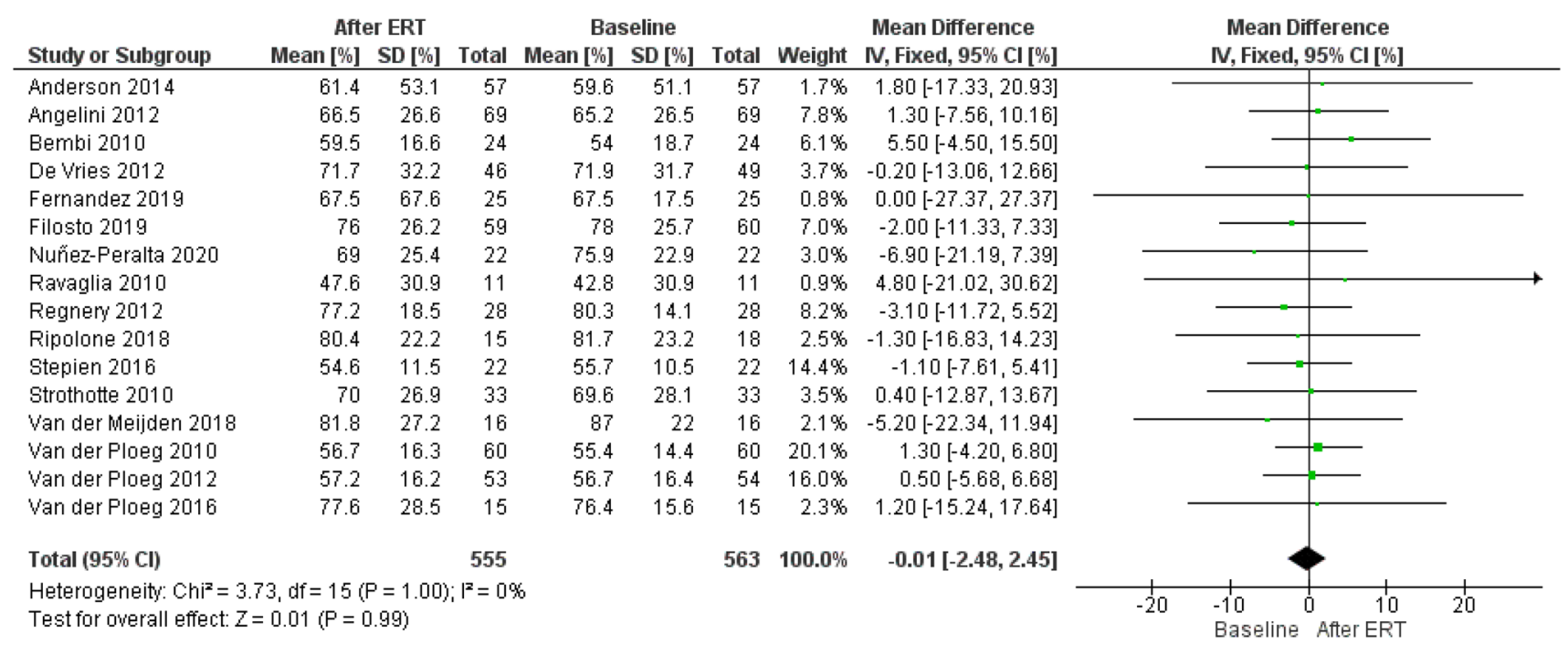

Fig. 4 Forest plot of effect of enzyme replacement therapy on respiratory function, as assessed by forced vital capacity 
Forest plot of stratified analysis demonstrated that after 19.5 months, patients on rhGAA did not show significant improvements in muscle strength even though the average shows an increase of approximately 0.07 points (95\%-CI $-0.05-0.19 ; p=0.24$ ) during the observed period, compared to muscle strength at baseline.

Assessment of publication bias using a funnel plot indicated symmetry as shown in Fig. 7.

Two of the included studies $[1,21]$ reported muscle strength results in two groups as composite QMT leg and arm scores (i.e., the average of the percentage of predicted scores for bilateral knee flexors and extensors and bilateral elbow flexors and extensors). To optimize the number of results for our search, both determinations were included for the analysis, which represents the same population.

\section{Discussion}

\section{Main findings}

Fourteen years after ERT became available, we conducted this systematic review to update the previous findings and to perform a meta-analysis on the efficacy of ERT in patients with LOPD. Outcomes of interest were walking distance, respiratory function, and muscle strength after begin of ERT compared to their baseline values. As the main result, we found that in patients with LOPD after ERT the walking distance significantly improved, however, the respiratory function and the muscle strength remained unchanged.

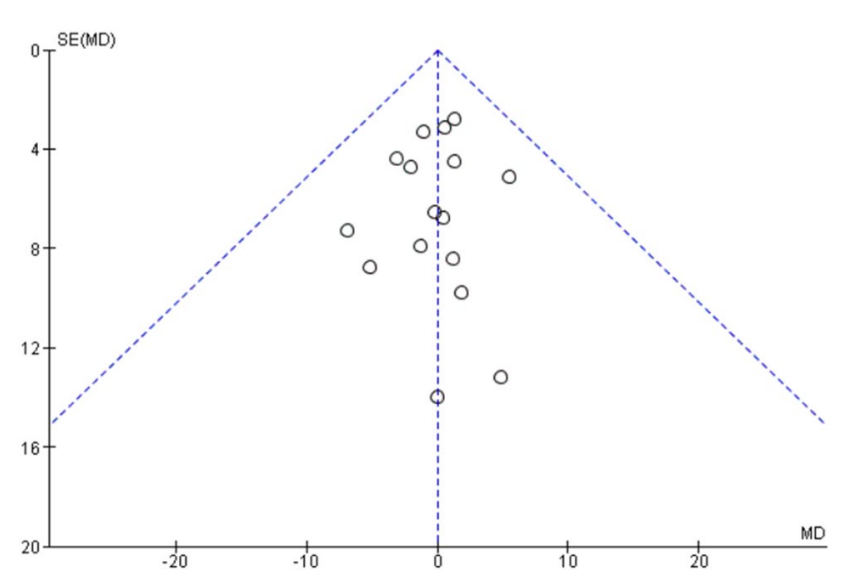

Fig. 5 Funnel plot of comparison of forced vital capacity

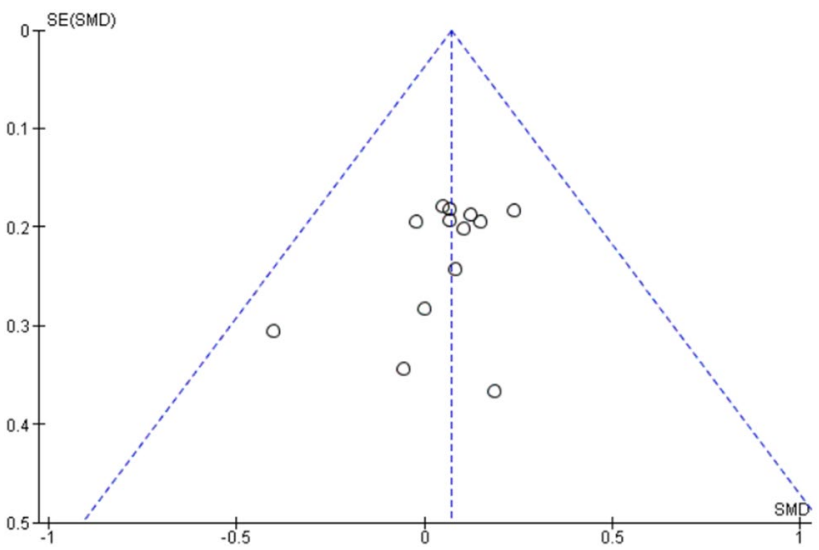

Fig. 7 Funnel plot of comparison of medical research council grading scale, quantitative muscles testing and quick motor function test

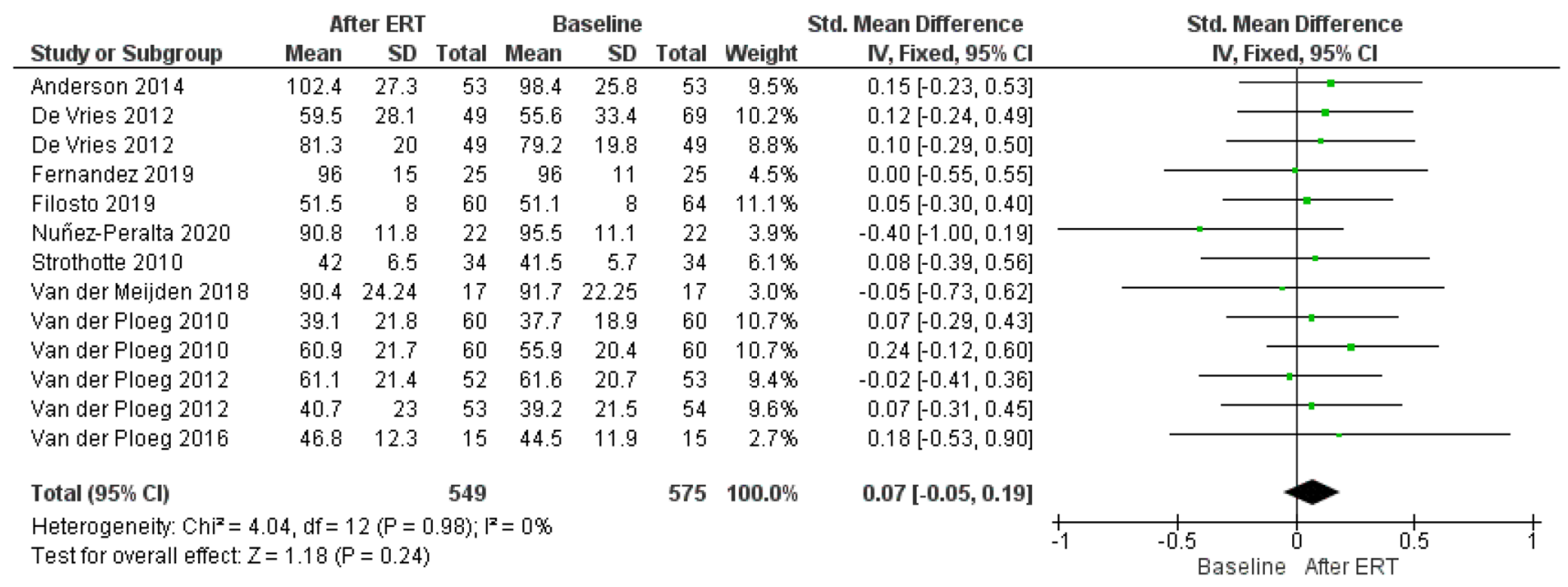

Fig. 6 Forest plot of effect of enzyme replacement therapy on muscle strength, as assessed by medical research council grading scale, quantitative muscles testing and quick motor function test 


\section{Results in context}

Güngör and co-workers reported a survival benefit under ERT [30]. In a recent review with 368 patients, ERT was associated with improved or stabilized muscular and respiratory functions [10]. More recently, a systematic review on survival and muscular function reported a nearly five-fold lower mortality rate. Furthermore, 6MWT improved over the first 20 months of therapy followed by a stabilization [11].

Regarding the FVC, Schoser and co-worked reported a rapid improvement of the respiratory function under ERT, followed by a slow regression to baseline over three-years period, while among untreated patients the respiratory function declined over time [11]. Similarly, our meta-analysis showed that the FVC of patients treated with ERT did not improve beyond 20.75 months. However, at an individual level, the response to treatment varied. The reasons for this variation in clinical response need further investigation, especially since respiratory failure represents the leading cause of death in patients with LOPD [1, 6, 7]. Phenotypic, and genotypic factors associated with LOPD might likely play a role. In addition, a diagnostic delay that may postpone an earlier begin of ERT might influence the treatment response. Since the deterioration of lung function is the major cause of mortality in patients with LOPD, even if ERT does not produce a significant improvement in FVC, it would have a substantial impact on patient survival, by preventing or slowing down the decline of the respiratory function.

In summary, the results of our meta-analysis confirm the findings of the previous systematic review. Unlike from the previous studies, however, we investigated a third outcome, the muscle strength. We decided to include also this endpoint because all together, 6-MWT, FVC, and muscle strength represent the first recommended functional assessment by the Swiss Guidelines of Pompe disease [31], in monitoring the patients, and are the same outcomes recommended by the European Pompe Consortium [12]. In the analysis, we included studies that evaluated the muscle strength using the MRC grading scale, the QMT and the QMFT, which are the most used tools to evaluate muscle strength in Europe [32] and they also were already used in previous reviews on patients with JOPD [13]. Additionally, we performed a meta-analysis to investigate the efficacy of ERT on muscle strength in patients with LOPD. In the identified studies, referring to 413 patients treated with ERT, the muscle strength did not increase significantly, despite a trend towards improvement of muscle strength was observed.

\section{Implications for research}

Phenotypic and genotypic factors might have a role in the prediction of the therapeutic response to ERT in patients with LOPD. In addition, the development of antibodies against ERT, which may reduce its effect could influence the efficacy of the therapy $[33,34]$. Further studies are needed to better identify these factors and, hence, patients who might benefit from the therapy. In fact, knowing which patients have a better response would allow a more efficient patient prioritizing and an optimizing of resources. Especially in countries with limited resources this might be of concern [35].

\section{Implications for practice}

Some studies have suggested that early initiation of ERT may improve the clinical response to the treatment $[1,17$, 36]. Given that, the American Association of Neuromuscular and Electrodiagnostic Medicine [37] recommends starting ERT in all symptomatic patients with a confirmed diagnosis. Similarly, the European Pompe Consortium [12] recommends initiating ERT in symptomatic patients who agree to regular treatment and monitoring, have residual skeletal and respiratory muscle function, and do not have another life-threatening illness in an advanced stage. However, to better identify the predictors of the therapeutic response, it is crucial to continue to collect data on cohorts of patients with LOPD. Furthermore, due to the growing interest for the use of neo-GAA as well as ERT combined with a chaperon in patients with LOPD, further studies are needed to verify the effectiveness of these new treatments [38].

\section{Strengths/Limitations}

Strengths of this review are that several new publications have been added compared to the previous systematic reviews, and at the same time, the overlap of patients between studies was taken into account. Furthermore, this work represents one of the few systematic reviews including a meta-analytic design referring specifically to patients with LOPD. Furthermore, muscle strength was included as an outcome of interest.

This review has also limitations. First, only a relatively small number of studies with a small number of patients have been included. However, this is not surprising given how rare LOPD is. To limit this bias, we included in the analysis only studies with more than 10 patients and we excluded clinical cases. Second, considering the variety of settings and designs of the included studies, results are susceptible to selection bias. Third, we limited the evaluation of the efficacy of ERT in patients with LOPD on 6MWT, FVC, and muscle strength. Some further outcomes, such as clinical improvement perceived by patients, measurements specifically developed to evaluate LOPD or at least more specific to neuromuscular disease, should also be considered (39). Finally, outcomes of interest in the included studies were evaluated at different follow-up times, from 6 months 
to 7 years after initiation of ERT, limiting the generalization of our findings.

\section{Conclusions}

In conclusion, this study updates previous literature reviews on the efficacy of ERT in patients with LOPD. It shows that in patients with LOPD treated with ERT, the walking distance significantly improved while the muscle strength only showed a non-significant improvement, though the respiratory function did not change. Only a few studies had an adequate methodological quality to be included in our metaanalysis; therefore, new clinical trials are necessary to obtain more robust results about the clear clinical benefit of ERT.

Authors' contributions Optional: please review the submission guidelines from the journal whether statements are mandatory.

Funding Open Access funding provided by Universität Zürich. Not applicable. (The funders played no role in the preparation of the manuscript or the decision to submit for publication.)

\section{Declarations}

Conflicts of interest The authors declare that there is no conflict of interest regarding the publication of this paper.

Ethical approval No ethical approval was needed. All studies in this review have been approved by the appropriate ethics committee and have therefore been performed in accordance with the ethical standards laid down in the 1964 Declaration of Helsinki and its later amendments.

Informed consent Data from previously published studies, in which informed consent was obtained were retrieved and analyzed.

Consent for publication All the authors of this manuscript have been included and we warrant that nobody who qualifies for authorship has been excluded. We agree to its submission to the Journal of Neurology and, if accepted, to its publication in this journal.

Open Access This article is licensed under a Creative Commons Attribution 4.0 International License, which permits use, sharing, adaptation, distribution and reproduction in any medium or format, as long as you give appropriate credit to the original author(s) and the source, provide a link to the Creative Commons licence, and indicate if changes were made. The images or other third party material in this article are included in the article's Creative Commons licence, unless indicated otherwise in a credit line to the material. If material is not included in the article's Creative Commons licence and your intended use is not permitted by statutory regulation or exceeds the permitted use, you will need to obtain permission directly from the copyright holder. To view a copy of this licence, visit http://creativecommons.org/licenses/by/4.0/.

\section{References}

1. van der Ploeg AT, Clemens PR, Corzo D, Escolar DM, Florence J, Groeneveld GJ et al (2010) A randomized study of alglucosidase alfa in late-onset Pompe's disease. N Engl J Med 362(15):1396-1406

2. Dasouki M, Jawdat O, Almadhoun O, Pasnoor M, McVey AL, Abuzinadah A et al (2014) Pompe disease: literature review and case series. Neurol Clin 32(3):751-776

3. Bembi B, Cerini E, Danesino C, Donati MA, Gasperini S, Morandi L et al (2008) Management and treatment of glycogenosis type II. Neurology 71(23 Suppl 2):S12-36

4. Pascual SIP (2009) Phenotype variations in early onset Pompe disease: diagnosis and treatment results with Myozyme. Adv Exp Med Biol 652:39-46

5. Patel TT, Banugaria SG, Case LE, Wenninger S, Schoser B, Kishnani PS (2012) The impact of antibodies in late-onset Pompe disease: a case series and literature review. Mol Genet Metab 106(3):301-309

6. van der Ploeg AT, Reuser AJJ (2008) Pompe's disease. Lancet Lond Engl 372(9646):1342-1353

7. van der Ploeg A, Carlier PG, Carlier R-Y, Kissel JT, Schoser B, Wenninger S et al (2016) Prospective exploratory muscle biopsy, imaging, and functional assessment in patients with late-onset Pompe disease treated with alglucosidase alfa: the EMBASSY Study. Mol Genet Metab 119(1-2):115-123

8. van der Meijden JC, Güngör D, Kruijshaar ME, Muir ADJ, Broekgaarden HA, van der Ploeg AT (2015) Ten years of the international Pompe survey: patient reported outcomes as a reliable tool for studying treated and untreated children and adults with non-classic Pompe disease. J Inherit Metab Dis 38(3):495-503

9. Kishnani PS, Corzo D, Leslie ND, Gruskin D, van der Ploeg A, Clancy JP et al (2009) Early treatment with alglucosidase alfa prolongs long term survival of infants with Pompe disease. Pediatr Res 66(3):329-335

10. Toscano A, Schoser B (2013) Enzyme replacement therapy in late-onset Pompe disease: a systematic literature review. J Neurol 260(4):951-959

11. Schoser B, Stewart A, Kanters S, Hamed A, Jansen J, Chan K et al (2017) Survival and long-term outcomes in late-onset Pompe disease following alglucosidase alfa treatment: a systematic review and meta-analysis. J Neurol 264(4):621-630

12. van der Ploeg AT, Kruijshaar ME, Toscano A, Laforêt P, Angelini C, Lachmann RH et al (2017) European consensus for starting and stopping enzyme replacement therapy in adult patients with Pompe disease: a 10-year experience. Eur J Neurol 24(6):768-e31

13. Milverton J, Newton S, Merlin T (2019) The effectiveness of enzyme replacement therapy for juvenile-onset Pompe disease: a systematic review. J Inherit Metab Dis 42(1):57-65

14. Moher D, Liberati A, Tetzlaff J, Altman DG, PRISMA Group (2009) Preferred reporting items for systematic reviews and metaanalyses: the PRISMA statement. PLoS Med 6(7):e1000097

15. Introduction to Meta-Analysis I Wiley [Internet]. Wiley.com. Available from: https://www.wiley.com/en-us/Introduction+to+ Meta+Analysis-p-9780470057247 (accessed 7 Oct 2020)

16. Ravaglia S, Pichiecchio A, Ponzio M, Danesino C, Saeidi Garaghani K, Poloni GU et al (2010) Changes in skeletal muscle qualities during enzyme replacement therapy in late-onset type II glycogenosis: temporal and spatial pattern of mass vs. strength response. J Inherit Metab Dis 33(6):737-745

17. Strothotte S, Strigl-Pill N, Grunert B, Kornblum C, Eger K, Wessig $C$ et al (2010) Enzyme replacement therapy with alglucosidase alfa in 44 patients with late-onset glycogen storage disease type 2: 12-month results of an observational clinical trial. J Neurol 257(1):91-97 
18. Bembi B, Pisa FE, Confalonieri M, Ciana G, Fiumara A, Parini R et al (2010) Long-term observational, non-randomized study of enzyme replacement therapy in late-onset glycogenosis type II. J Inherit Metab Dis 33(6):727-735

19. Angelini C, Semplicini C, Ravaglia S, Bembi B, Servidei S, Pegoraro E et al (2012) Observational clinical study in juvenileadult glycogenosis type 2 patients undergoing enzyme replacement therapy for up to 4 years. J Neurol 259(5):952-958

20. de Vries JM, van der Beek NA, Hop WC, Karstens FP, Wokke JH, de Visser M et al (2012) Effect of enzyme therapy and prognostic factors in 69 adults with Pompe disease: an open-label singlecenter study. Orphanet J Rare Dis 26(7):73

21. van der Ploeg AT, Barohn R, Carlson L, Charrow J, Clemens PR, Hopkin RJ et al (2012) Open-label extension study following the Late-Onset Treatment Study (LOTS) of alglucosidase alfa. Mol Genet Metab 107(3):456-461

22. Regnery C, Kornblum C, Hanisch F, Vielhaber S, Strigl-Pill N, Grunert B et al (2012) 36 months observational clinical study of 38 adult Pompe disease patients under alglucosidase alfa enzyme replacement therapy. J Inherit Metab Dis 35(5):837-845

23. Anderson LJ, Henley W, Wyatt KM, Nikolaou V, Waldek S, Hughes DA et al (2014) Effectiveness of enzyme replacement therapy in adults with late-onset Pompe disease: results from the NCS-LSD cohort study. J Inherit Metab Dis 37(6):945-952

24. Stepien KM, Hendriksz CJ, Roberts M, Sharma R (2016) Observational clinical study of 22 adult-onset Pompe disease patients undergoing enzyme replacement therapy over 5years. Mol Genet Metab 117(4):413-418

25. Ripolone M, Violano R, Ronchi D, Mondello S, Nascimbeni A, Colombo I et al (2018) Effects of short-to-long term enzyme replacement therapy (ERT) on skeletal muscle tissue in late onset Pompe disease (LOPD). Neuropathol Appl Neurobiol 44(5):449-462

26. van der Meijden JC, Kruijshaar ME, Harlaar L, Rizopoulos D, van der Beek NAME, van der Ploeg AT (2018) Long-term follow-up of 17 patients with childhood Pompe disease treated with enzyme replacement therapy. J Inherit Metab Dis 41(6):1205-1214

27. Fernández-Simón E, Carrasco-Rozas A, Gallardo E, GonzálezQuereda L, Alonso-Pérez J, Belmonte I et al (2019) Study of the effect of anti-rhGAA antibodies at low and intermediate titers in late onset Pompe patients treated with ERT. Mol Genet Metab 128(1-2):129-136

28. Filosto M, Cotti Piccinelli S, Ravaglia S, Servidei S, Moggio M, Musumeci O et al (2019) Assessing the role of anti rh-GAA in modulating response to ERT in a late-onset pompe disease cohort from the Italian GSDII study group. Adv Ther 36(5):1177-1189

29. Nuñez-Peralta C, Alonso-Pérez J, Llauger J, Segovia S, Montesinos P, Belmonte I et al (2020) Follow-up of late-onset Pompe disease patients with muscle magnetic resonance imaging reveals increase in fat replacement in skeletal muscles. J Cachexia Sarcopenia Muscle 11(4):1032-1046. https://doi.org/10.1002/jcsm. 12555

30. Güngör D, Kruijshaar ME, Plug I, D’Agostino RB, Hagemans MLC, van Doorn PA et al (2013) Impact of enzyme replacement therapy on survival in adults with Pompe disease: results from a prospective international observational study. Orphanet J Rare Dis 27(8):49

31. Hundsberger T, Rohrbach M, Kern L, Rösler KM (2013) Swiss national guideline for reimbursement of enzyme replacement therapy in late-onset Pompe disease. J Neurol 260(9):2279-2285

32. Schoser B, Laforêt P, Kruijshaar ME, Toscano A, van Doorn PA, van der Ploeg AT et al (2015) 208th ENMC International Workshop: Formation of a European Network to develop a European data sharing model and treatment guidelines for Pompe disease Naarden, The Netherlands, 26-28 September 2014. Neuromuscul Disord NMD 25(8):674-678

33. Kishnani PS, Goldenberg PC, DeArmey SL, Heller J, Benjamin D, Young $S$ et al (2010) Cross-reactive immunologic material status affects treatment outcomes in Pompe disease infants. Mol Genet Metab 99(1):26-33

34. van Gelder CM, Hoogeveen-Westerveld M, Kroos MA, Plug I, van der Ploeg AT, Reuser AJJ (2015) Enzyme therapy and immune response in relation to CRIM status: the Dutch experience in classic infantile Pompe disease. J Inherit Metab Dis 38(2):305-314

35. Muranjan M (2014) Enzyme replacement therapy for lysosomal storage disorders in India. Mol Cytogenet 7(1):I29

36. Kishnani PS, Nicolino M, Voit T, Rogers RC, Tsai AC-H, Waterson J et al (2006) Chinese hamster ovary cell-derived recombinant human acid alpha-glucosidase in infantile-onset Pompe disease. J Pediatr. 149(1):89-97

37. Cupler EJ, Berger KI, Leshner RT, Wolfe GI, Han JJ, Barohn RJ et al (2012) Consensus treatment recommendations for late-onset Pompe disease. Muscle Nerve 45(3):319-333

38. Davison JE (2020) Advances in diagnosis and management of Pompe disease. J Mother Child 24(2):3-8

39. Lachmann R, Schoser B (2013) The clinical relevance of outcomes used in late-onset Pompe disease: can we do better? Orphanet $\mathrm{J}$ Rare Dis 8(1):160 